\title{
The Emergence of a Coupled Quantum Dot Array in a Doped Silicon Nanowire Gated by Ultrahigh Density Top Gate Electrodes ${ }^{\dagger}$
}

\author{
Ke Xu, ${ }^{\ddagger}$ Jonathan E. Green, J. R. Heath, ${ }^{*,+}$ F. Remacle, ${ }^{\S, \|}$ and R. D. Levine ${ }^{*, \S, \perp}$ \\ The Division of Chemistry and Chemical Engineering, California Institute of Technology, \\ MC 127-72, Pasadena, California 91125, The Fritz Haber Research Center for Molecular Dynamics, \\ The Hebrew University of Jerusalem, Jerusalem 91904, Israel, Département de Chimie, B6c, \\ Université de Liège, B4000 Liège, Belgium, and Department of Chemistry and Biochemistry, \\ The University of California Los Angeles, Los Angeles, California 90095
}

Received: February 16, 2007; In Final Form: May 1, 2007

\begin{abstract}
The electrical characteristics of Si nanowire gated by an array of very closely spaced nanowire gate electrodes are experimentally determined and theoretically modeled. Qualitative and quantitative changes in the transport characteristics of these devices, as a function of gate-array voltage, are described. Experiments are reported for two widths of Si nanowires, 40 and $17 \mathrm{~nm}$, and for a varying number of gate electrodes, all spaced at a pitch of $33 \mathrm{~nm}$. We find that these top nanowire gate electrodes can be utilized to locally deplete the carriers in the underlying $\mathrm{Si}$ nanowire and thus define an array of coupled quantum dots along the nanowire. Reproducible Coulomb blockade is observed, and clear diamond features are obtained when the conductance is plotted in the plane of the source-drain and gate voltages. The regularity of the diamond diagrams is imposed by the regularity of the SNAP top gate electrodes. Model computations of the electronic structure starting from a tight-biding Hamiltonian in the atomic basis suggest that the control made possible by the top gate voltage induces the emergence (and reversible submergence) of a coupled quantum dot structure in an otherwise homogenously doped Si nanowire.
\end{abstract}

\section{Introduction and Experimental Background}

Sufficiently small $(<100 \mathrm{~nm})$ particles can exhibit charge quantization-like natural atoms. Such "artificial atom"1,2 finite fermion systems are often called quantum dots (QDs), referring to the quantum confinement in all three spatial dimensions. The charge transport properties of QD systems are characterized by single-electron charging and resonant tunneling through the quantized energy levels of the QDs. ${ }^{1-4}$ A host of lithographically defined $^{1-4}$ and chemically synthesized ${ }^{5,6}$ QD-based devices have been electronically characterized. As single-electron devices, QDs represent an ultimate limit. ${ }^{7,8}$ They can also serve as building blocks to form more complex architectures with intriguing and novel properties. For example, we reported a reversible metal-insulator transition in silver QD monolayers. ${ }^{9}$ In fact, it is the theoretical models developed for that system ${ }^{10-12}$ that provide the starting framework for much of the theory discussed here. Coupled QD systems, ${ }^{13-16}$ in particular double QDs, ${ }^{17,18}$ have also been investigated, often as platforms for the realization of solid-state quantum bits. ${ }^{19}$ For these experiments, gate electrodes are utilized to both spatially confine and to control the coupling between the QDs.

Lithographically patterned (field-confined) QDs are relatively large structures, typically on the order of $100 \mathrm{~nm}$ or so. While this implies the need for ultralow temperature measurements, such QDs are readily integrated into device platforms, and

\footnotetext{
† Part of the special issue "Richard E. Smalley Memorial Issue".

* To whom correspondence should be addressed. E-mail: (J.R.H.) heath@caltech.edu or (R.D.L.) rafi@fh.huji.ac.il

$\doteqdot$ California Institute of Technology.

$\S$ The Hebrew University of Jerusalem.

"Université de Liège.

$\perp$ The University of California Los Angeles.
}

electrical contacts to the QDs are intrinsically established. By contrast, chemically synthesized (spatially confined) QDs, while challenging to incorporate into device platforms, are significantly smaller and so are characterized by much larger energy level spacings and can be interrogated at higher temperatures. A compromise between these two classes of devices are QDs defined within single-wall carbon nanotubes ${ }^{20}$ and semiconductor nanowires (NWs). ${ }^{21,22}$ Here, the size of the QD is defined by tunnel barriers at the source and drain contacts to a NWbased field-effect transistor (FET). Smaller QDs have been achieved by utilizing intrinsic ${ }^{23,24}$ or induced ${ }^{25}$ defects in nanotubes or barrier heterostructures intentionally introduced during the axial growth of the NWs. ${ }^{26,27}$ Nevertheless, gated measurements of coupled QDs within these NW systems have involved relatively large gate electrodes and electrode spacings, and so the interdot coupling energies are low. ${ }^{28-30}$ Narrower and more closely spaced gates, coupled with true-size confined QDs, would serve to increase the coupling strength. An experimental demonstration and a theoretical description of such devices constitute the focus of this paper.

We report here on experimental and theoretical investigations of Si NW-based coupled QD systems. We investigate both 40 and $17 \mathrm{~nm}$ wide Si NWs, with gate electrodes comprised of a closely spaced array of metal NWs. The gate electrode width and pitch (periodicity) are 17 and $33 \mathrm{~nm}$, respectively, and between 3 and 10 gate electrodes are utilized, depending upon the device.

Silicon [or silicon-on insulator (SOI)] is, in principle, an attractive material for QD experiments due to its highly developed fabrication technology and the potential integration of QD devices with conventional electronics circuits. ${ }^{8,31}$ Previous approaches toward building QD systems on SOI substrates have 


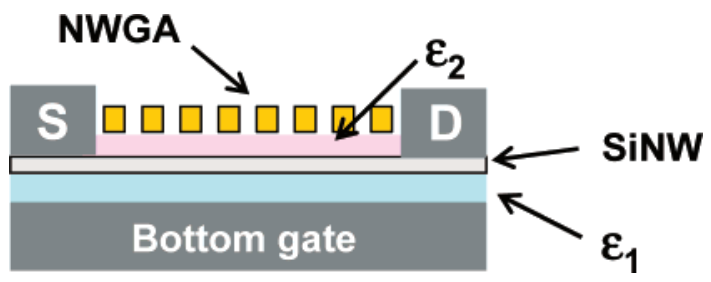

Figure 1. Drawing of a SiNW QD device, contacted by source (S) and drain (D) electrodes, and with two types of gates. The bottom gate, separated from the NW by dielectric $\epsilon_{1}$, uniformly affects the entire SiNW. The NWGA, separated from the NW by $\epsilon_{2}$, partitions the NW into a series of coupled QDs.

mainly focused on obtaining ultrathin NWs from lithography followed by etching procedures to reduce the NW width. ${ }^{32-37}$ Single-electron effects have been observed in these devices, but the results disagree with the designed device geometry; the etching processes introduce defects and roughness that, in turn, lead to the random formation of QDs within the nanostructure and irreproducible transport characteristics. For example, Coulomb blockade diamond diagrams, a signature of single-electron charging, are typically not obtainable because the change of signal in consecutive scans can be much larger than the gate influence. ${ }^{37}$ Electrostatically defined systems have resulted in highly controllable and reproducible coupled double QDs along $\mathrm{NWs},{ }^{30}$ but the lithography step employed to pattern the gate electrodes limits the spacing between the gates (and hence the length of each QD) to be relatively large $(\sim 100 \mathrm{~nm})$. This leads to very small charging energy scales (on the order of $1 \mathrm{meV}$ ) and correspondingly low coupling strengths for the QDs. ${ }^{30}$

We utilized the superlattice NW pattern transfer (SNAP) method $^{38,39}$ to produce high-quality Si NWs as a template for investigating coupled QDs. We also utilized SNAP to pattern an array of high-density metal NW top gate electrodes. This novel combination of geometric confinement from the width of the Si NW, the highly regular NWs that are formed using the SNAP method, and the electric field confinement from the ultrahigh-density top gates allows for the controlled formation of a concatenated array of ultrasmall $(17 \mathrm{~nm})$ QDs in series along the length of the Si NW for the first time. As a result, reproducible Coulomb blockade is observed with much larger energy scales (on the order of 10-50 meV), and full characterization of devices is achieved at readily attainable liquid helium temperatures $(\sim 4 \mathrm{~K})$. The small sizes and close proximity of the individual QDs mean that, in contrast to lithographically defined systems, the ratio of the size variation from dot-to-dot to the actual dot size is a relatively large number. This implies that disorder may dominate the observed transport data. In fact, we find that the regularity of the diamond diagrams is closely related to the regularity of the top gate electrodes. The gate capacitance of each QD, as calculated from the diamond diagrams, is in agreement with the gate capacitance calculated from the geometry of each QD defined by top gate electrodes. In addition, grouping of Coulomb blockade diamonds resulted from the coupling of the QDs is revealed. The QD size effect is also examined by varying the width of the underlying Si NWs. These results clearly demonstrate that coupled QDs in series have been defined along the underlying Si NW by the ultrahigh-density SNAP top gate electrodes.

\section{NW QD Systems}

A schematic of a doubly gated NW/QD device is presented in Figure 1. There are two types of gate electrodes shown-a bottom gate and a top NW gate array (NWGA). While the label "bottom gate" does have a geometrical significance (it is

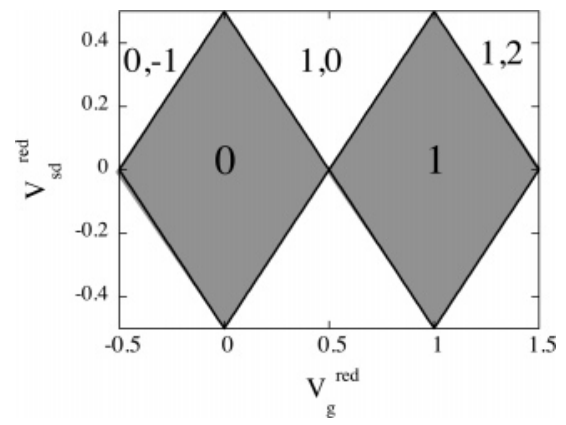

Figure 2. Stability map for a QD with $N=0$ and $N=1$ electrons, plotted as a function of the $V_{\mathrm{g}}$ and $V_{\mathrm{sd}}$ in reduced units. $V_{\mathrm{g}}^{\mathrm{red}}=C_{\mathrm{g}} V_{\mathrm{g}} / e$ corresponds to the number of electrons on the dot, and $V_{\mathrm{sd}}^{\mathrm{red}}=V_{\mathrm{sd}} C_{\mathrm{T}} /$ $2 e$.

underneath the NW), it is not the geometry but rather the different roles that the two gates perform that is important. Although the experimental measurements presented here utilized only the NWGA, we have reported bottom- (or single-) gate type measurements on similar SiNWs elsewhere. ${ }^{40,41}$ With the bottom gate voltage $\left(V_{\mathrm{BG}}\right)$ set to 0 , the source drain voltage $\left(V_{\text {sd }}\right)$ can drive a current through the NW. The NW is, by the nature of its size, a quantum-confined system at low temperature, provided the tunnel barriers at the source and drain contacts are of high enough energy for confinement. A simple model computation shows that the current-voltage curve has a staircase structure with rather narrowly spaced stairs and with an energy width that is expected for single particle spacings in a finite wire of doped $\mathrm{Si}^{22}$ A finite value of $V_{\mathrm{BG}}$ would shift the energy levels of the entire NW, which leads to the familiar Coulomb diamond structures in a plot of the source-drain conductance $\left(\partial I / \partial V_{\mathrm{sd}}\right)$ vs $V_{\mathrm{BG}}$, as shown in Figure 2, until all of the charge carriers are depleted by the gate and the NW becomes insulating. The $\sim 1 \mathrm{meV}$ energy scale, corresponding to the narrow energy spacings, would be difficult to observe at liquid helium temperature.

Now, we turn to the NWGA. Consider the influence of a finite $V_{\mathrm{NWGA}}$ on the NW conductance. The simple picture described above undergoes a qualitative change. As the gate voltage is increased, a new electronic structure emerges in a manner reminiscent of emergent phenomena in general. ${ }^{42}$ Those $\mathrm{Si}$ atoms that are under any one particular top NW self-organize as a QD and become decoupled from those Si atoms on their left and right that are not under the gate electrode. The Si atoms under adjacent top electrodes also respond collectively, and moreover, the dots that are defined by neighboring top electrodes are found to be coupled. A finite value of $V_{\mathrm{NWGA}}$ is required for the QD structure to emerge. Past that threshold, increasing $V_{\text {NWGA }}$ increases the number of discrete quantum levels of the dots. The model that we use to describe this emergence is simplistic: a linear array of exchange-coupled Si atoms with excess charges due to doping. Nevertheless, this model suffices to capture the emergence of a QD structure in the gated $\mathrm{Si}$ NW. Furthermore, the computed electrical response is in agreement with the features observed experimentally. We use a simple model also because plotting Coulomb diamond maps as a function of $V_{\text {NWGA }}$ and of $V_{\text {sd }}$ is much more demanding theoretically, since at each value of $V_{\text {NWGA }}$ we have a different system. In other words, one can loosely say that for finite values of $V_{\mathrm{BG}}$, variation of that gate voltage allows one to do spectroscopy on the same system but at different levels of excitation. If that single gate is replaced with an array of NW gate electrodes, the very electronic structure of the system is fundamentally changed. 
The evidence for the pattern of QDs imposed by the NWGA on the SiNW is both experimental and theoretical. The observable signature of a three terminal device with a discrete quantal level structure consists of Coulomb "diamond" structures observed when the conductance, $\partial I / \partial V_{\text {sd }}$, is plotted as a function of the source drain $\left(V_{\mathrm{sd}}\right)$ and gate voltages. ${ }^{1-4} \mathrm{~A}$ Coulomb diamond plot is easy to interpret in a simplistic theory where the potential difference between the source and the drain electrodes gives rise to a "Fermi window" with sharp boundaries (at low temperature). An energy level is either within this window or it is not. Thus, the current is a step function of $V_{\text {sd. }}$. The derivative of the step is a $\delta$ function. If both $V_{\mathrm{sd}}$ and the back gate are allowed to vary, then the lines of maximal derivative are at an angle to the axes as shown in Figure 2.

When we interpret the diamond structure as discrete levels moving into or out of the Fermi window defined by $V_{\text {sd }}$, it naturally follows that the structure can be observed not only for lithographically defined ${ }^{1-4}$ QDs and dots along a wire ${ }^{21,22}$ but also for chemically synthesized colloidal dots, ${ }^{10,12}$ ordinary molecules, ${ }^{43-45}$ and carbon nanotubes. ${ }^{20}$ The diamond structure is best seen when the coupling to the left and right electrodes is about equal and weak enough so that the device can be charged consecutively, one charge at a time. When there are many coupled dots, their discrete valence levels split into a band of states. For an array of only a few dots, the diamonds develop a fine structure, exemplified quite early by model computations. ${ }^{46}$ For a later discussion, see refs 14,15 , and 28 . We show, by both experiment and theory, that a similar structure is possible when the variables are $V_{\text {sd }}$ and $V_{\text {NWGA }}$. As already noted (Figure 1), a back gate affects the entire wire in a similar way while a top gate array has gaps between the NW electrodes and no potential is experienced under these gaps.

In the model computations, the dots emerge as sets of localized states, one set of states under each gate electrode. The number of levels in the set is found to increase as the gate voltage increases. It is this set of localized states that we identify as the QD. The wave function of the array at an energy corresponding to a level in a set is actually a superposition of wave functions; each component (= a level of a QD) is localized in its own region and with a negligible weight on the $\mathrm{Si}$ atoms that separate the different dots and act as bridges between them. One can think of the coupling of the different QDs as a superexchange ${ }^{47}$ because the energy of the empty level (the lowest unoccupied atomic orbital) of those $\mathrm{Si}$ atoms that are not gated is higher than the energies of the levels of the QDs. As expected for superexchange, bridge-coupled subsystems, the wave function practically vanishes on the bridges. ${ }^{47}$ The model computations also suggest that by gating different dots by using different voltages on the various NWs comprising the NWGA (our eventual experimental goal), it should be possible to achieve a very flexible electrical response.

\section{Theoretical Model}

The model that we use to describe the conductance is based on a working hypothesis that has been formulated on the basis of our earlier joint work on the conductivity of arrays of metallic colloidal QDs. ${ }^{10-12} \mathrm{We}$ validate the working hypothesis by comparing the theoretical predictions and the measured results. Comparing to equivalent circuit analysis ${ }^{18}$ in which QDs are assumed to already have formed by local gates, our model starts from a Hamiltonian description on the level of $\mathrm{Si}$ atoms and derives results equivalent to that phenomenological model and shows how the dot structure emerges from an originally homogeneous NW, without having been artificially put in at the beginning of modeling.
Skipping over all but the most essential details, the theoretical background is as follows: In the regime where the array of metallic dots is compressed so that adjacent dots are strongly coupled, we found that the relevant dimensionless coupling parameter that fully describes the physics is that suggested by Anderson $^{48,49}$ for disordered systems

$$
\chi=\frac{\Delta \alpha}{\beta}
$$

Here, $\Delta \alpha$ is the mean difference in the ionization potential of two (adjacent) dots and $\beta$ is the exchange coupling. The strong coupling regime is one where the exchange coupling $\beta$ is larger than the Coulomb charging energy. The difference in the ionization potentials of adjacent dots arises because the chemical synthesis produces colloidal QDs with a size distribution that has a finite width, and because of the confinement, the ionization potential of the dot varies (inversely) with its size.

For effectively strong dot-dot coupling $(\chi<1)$, the wavefunctions are delocalized and the array is conducting. For $\chi>$ 10, as suggested by Anderson, the wave functions are localized, and different wavefunctions are localized about different dots in the array as determined by the particular size of the individual dots. For the geometrically ordered but electronically disordered arrays ${ }^{50,51}$ we find that there is an intermediate coupling regime $(10>\chi>1)$, where the wavefunctions are delocalized over a finite domain, which is smaller than the entire array. In this regime, there can be wide fluctuations in the conductivity. To describe the essential physics of the gating of $p$ type Si NWs, we propose a tight binding Hamiltonian for a one-dimensional array of $\mathrm{Si}$ atoms. This is similar to the model used to explain the experimental results of ref 22 for $n$ type doping. For the dimensions of a four top gate device, we estimate that there are about $N=330 \mathrm{Si}$ atoms between the source and the drain electrodes. We couple the $\mathrm{Si}$ atoms by an exchange coupling that is estimated from the effective mass for doped Si. Unlike the case of colloidal dots that are variable in size, a tight binding Hamiltonian for an ordinary wire of atoms can be diagonalized analytically. As is known, the eigenvalues form a band and the corresponding eigenstates are delocalized all along the wire. In the presence of a finite $V_{\text {sd }}$, the uncoupled energies of the $\mathrm{Si}$ atoms shift in a systematic way. In the experiments reported here, $V_{\mathrm{sd}}$ is much below the width of the bands so that its role is small, and qualitatively, the wave functions are delocalized because the coupling parameter $\chi$ is very small (for $V_{\mathrm{NWGA}}=$ $0)$.

The picture of delocalized states changes in an essential way when a finite $V_{\text {NWGA }}$ is imposed. The ionization energies of those $\mathrm{Si}$ atoms that are under a gate electrode shift, down or up depending on the sign of the voltage, with the result that these atoms are no longer in resonance with the other nongated $\mathrm{Si}$ atoms. We designate the top gate-imposed difference in ionization energy by $\Delta \alpha$. Varying $V_{\text {NWGA }}$ varies $\Delta \alpha$, and it is thereby possible to change the value of the effective coupling parameter $\chi$ over the range from delocalized, $\chi<1$, to strongly localized, $\chi>10$. It is this variation that we explore in the model computations.

\section{Experimental Section}

The fabrication process flow is shown in Figure 3, and scanning electron microscopy micrographs of devices in various stages of fabrication are presented in Figure 4. The starting structures are single-crystal Si NWs defined by patterning a SOI substrate ( $p$ type, $\sim 5 \times 10^{-18} \mathrm{~cm}^{-3}$, Simgui, Shanghai, China). The larger SiNWs (40 nm wide, $30 \mathrm{~nm}$ thick) were patterned 

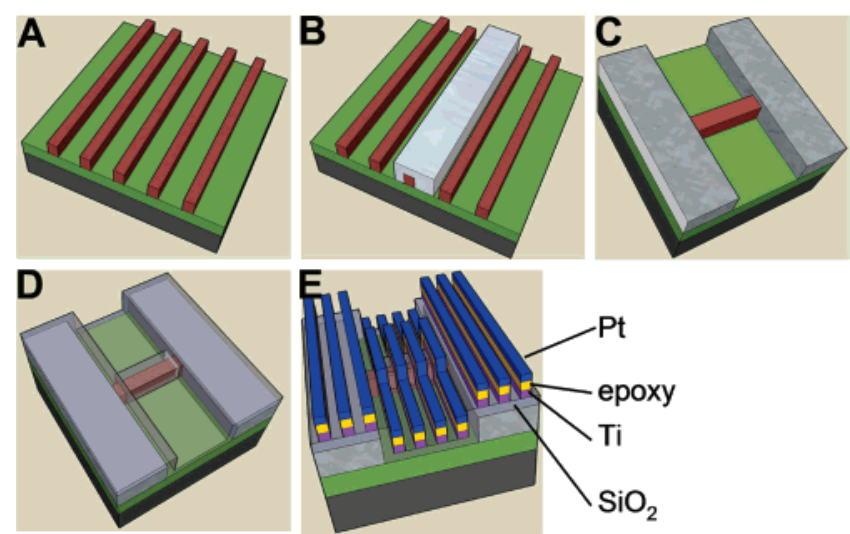

Figure 3. Process flow for the fabrication of a SiNW-based coupled QD device. (A) An array of $\sim 17 \mathrm{~nm}$ wide SiNWs with $50 \mathrm{~nm}$ spacing is prepared from a SOI using SNAP. (B) An EBL-defined, $\sim 50 \mathrm{~nm}$ wide aluminum mask is put down to protect one single SiNW. (C) The single SiNW is sectioned out and contacted with source/drain leads. (D) $\mathrm{A} \mathrm{SiO}_{2}$ layer is deposited on top as a dielectric layer. (E) An array of ultrahigh-density titanium NWs is fabricated on top of the dielectric layer perpendicular to the single SiNW, serving as NWGA. Note the titanium NWs over the source and drain leads are not expected to affect the transport properties of the device: Metals are naturally nongateable.

using electron-beam lithography (EBL), while the smaller NWs (17 $\mathrm{nm}$ wide, $30 \mathrm{~nm}$ thick) were prepared using the SNAP method. ${ }^{38,39}$ For both classes of NWs, the doping was accomplished using thermal diffusion of dopants from a spin-on dopant (Boron A, Filmtronics, Butler, PA). This process generates a gradient of dopant atoms through the thickness of the SOI wafer, effectively limiting the conducting part of the SiNW to the top $10 \mathrm{~nm}$ of its $30 \mathrm{~nm}$ thickness. ${ }^{52}$ Metallic contacts (Ti/Pt, 20/30 nm) were established to the SiNWs to generate source-drain channels that varied in length from 100 to $300 \mathrm{~nm}$. Thereafter, a high-density array of titanium gate electrodes (gate electrode width $\sim 17 \mathrm{~nm}$; pitch $=33 \mathrm{~nm}$ ) was fabricated on top of a $\mathrm{SiO}_{2}(\sim 15 \mathrm{~nm})$ dielectric layer perpendicular to the underlying SiNW (Figure 3) using the SNAP technique. The number of gate electrodes was varied from device to device: The smallest number was 3, and the largest number was $\sim 10$. These gate electrodes served to define the QDs within the underlying SiNWs. All gate electrodes were shorted to a larger contact control: Independent control of the different gate electrodes, which would constitute a nontrivial fabrication procedure, ${ }^{52}$ was not attempted for this initial investigation. Our eventual goal is, however, to achieve independent voltage control over the different gate electrodes and to thus control the coupling between the QDs. ${ }^{53,54}$ More details of the device fabrication are included in the Supporting Information.

\section{Experimental Results}

Experiments were first carried out on devices with an e-beam defined $\sim 40 \mathrm{~nm}$ wide underlying Si NW. Figure 5a shows such a device with about four top gate electrodes across the underlying Si NW, with modest disorder of that NWGA in the SiNW region. Even for devices in which the NWGA exhibited significant disorder, reproducible Coulomb blockade diamond diagrams were readily measured. Such reproducibility is demonstrated in the $\partial I / \partial V_{\text {sd }}-V_{\text {NWGA }}-V_{\text {sd }}$ plots of Figure $5 b, c$. The comparable sizes of the diamonds suggest the formation of QDs of comparable sizes along the NW, while irregularities of the diamonds are attributed to the inevitable small variation of the sizes of the QDs due to the irregularity of the NWGA.

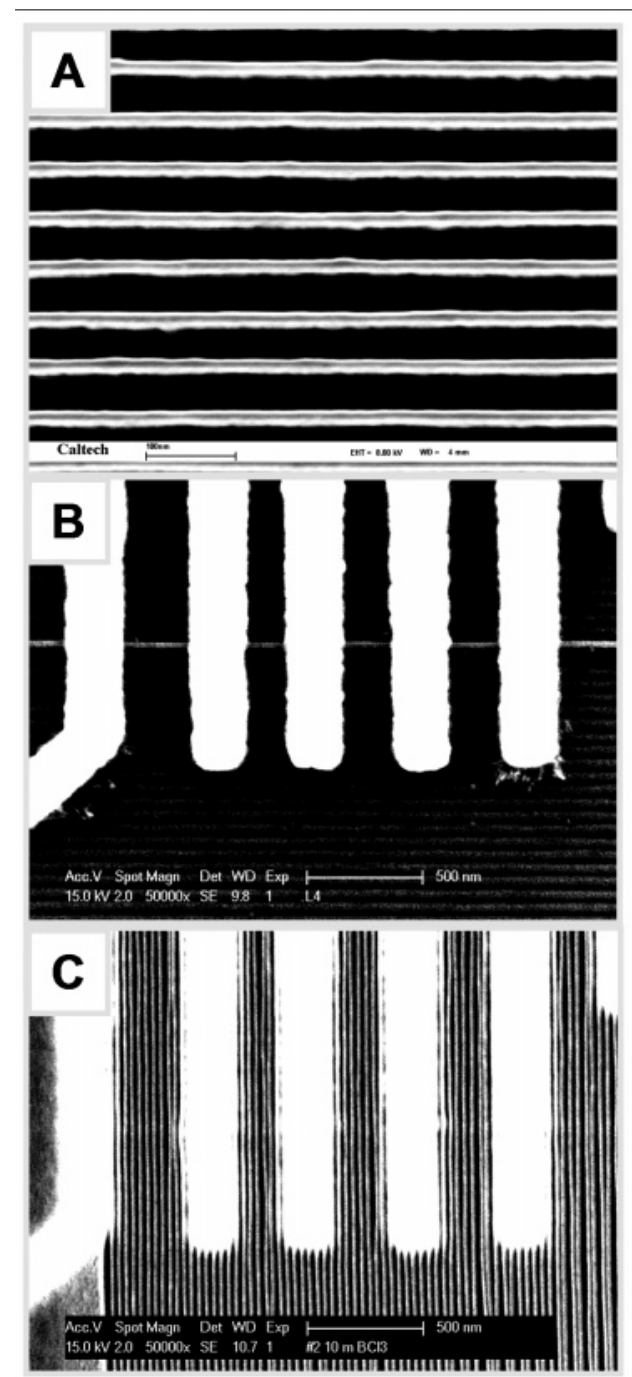

Figure 4. Scanning electron micrographs of three representative stages for the fabrication of devices with SNAP defined underlying SiNWs. (A) An array of $\sim 17 \mathrm{~nm}$ wide SiNWs with $50 \mathrm{~nm}$ spacing fabricated from a SOI using SNAP, corresponding to Figure 3A. Scale bar, 100 nm. (B) Five Ti/Pt contacts made to one single SiNW sectioned out from the array. Scale bar, $500 \mathrm{~nm}$. (C) The same structure after the dielectric layer and NWGA are fabricated on top, resulting in four full devices each corresponding to the structure in Figure 3E, with different source/drain distance and hence different number of top gate NWs.

Another $40 \mathrm{~nm}$ wide device with well-ordered NWGA is reported in the Supporting Information (Figure S1), and diamonds of similar energy scales were observed on that device as well. For comparison, we also performed measurements on another $40 \mathrm{~nm}$ wide device with a significantly more disordered NWGA, as shown in the Supporting Information (Figure S2). Reproducible Coulomb blockade was again observed for this device, with diamond-like features in the $\partial I / \partial V_{\mathrm{sd}}-V_{\mathrm{NWGA}}-$ $V_{\text {sd }}$ plot. In contrast to the results of Figure 5 and Figure $\mathrm{S} 1$, however, the sizes of the diamonds in this "disordered" device varied significantly, and diamond structures exhibited significant overlap with one another. These results were suggestive that QDs of varying sizes had been defined in series along the silicon $\mathrm{NW}$, in agreement with the observed structure of that specific device.

The properties of the QDs in these $40 \mathrm{~nm}$ wide devices can be obtained from the Coulomb blockade diamond diagrams as shown in Figure 5, Figure S1, and Figure 7.3,4,22 The addition energy $E_{\text {add }}$ can be directly measured from the maximum in $V_{\text {sd }}$ for the conductance gap of the diamonds, $\Delta V_{\mathrm{sd}} \sim 10 \mathrm{mV}$. This 


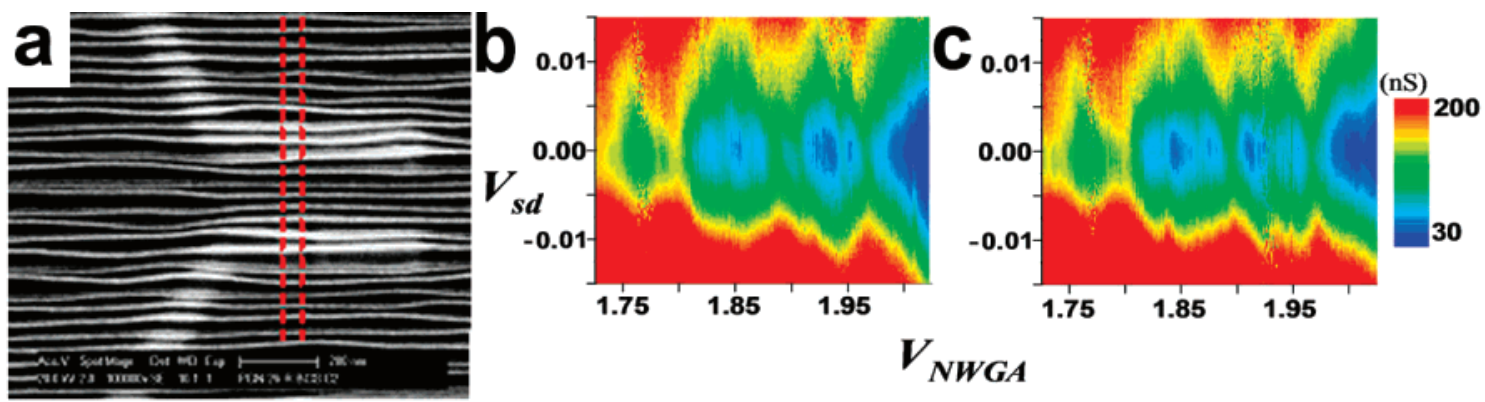

Figure 5. (a) Scanning electron micrograph of a device with an e-beam defined $\sim 40 \mathrm{~nm}$ wide underlying Si NW and some wandering of top gate electrodes. The contour of the underlying Si NW is highlighted with red dashed lines. Scale bar, $200 \mathrm{~nm}$. (b) $\partial I / \partial V_{\mathrm{sd}}-V_{\mathrm{NWGA}}-V_{\mathrm{sd}}$ plot of the device recorded at $4.3 \mathrm{~K}$, obtained when scanning the gate voltage from low to high. (c) The same plot obtained when scanning the gate voltage from high to low.
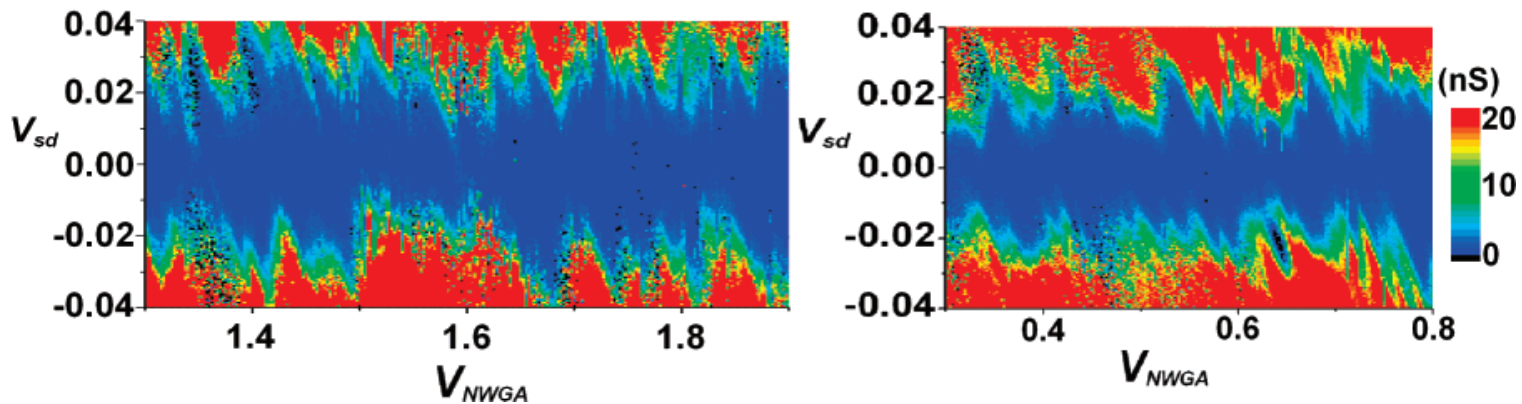

Figure 6. $\partial I / \partial V_{\text {sd }}-V_{\text {NWGA }}-V_{\text {sd }}$ plot of two devices with SNAP-defined underlying Si NWs, measured at $4.5 \mathrm{~K}$. Left plot: Measured from a device with a $\sim 17 \mathrm{~nm}$ wide underlying Si NW (Figure $4 \mathrm{c}$ ). Right plot: Measured from a device with a $\sim 20 \mathrm{~nm}$ wide underlying Si NW (Figure S3).

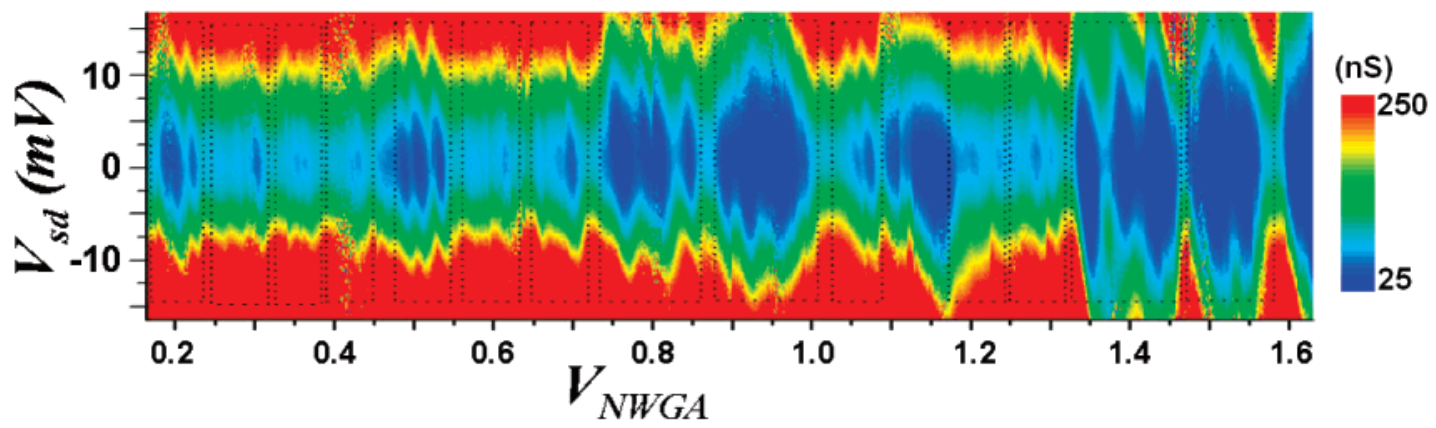

Figure 7. $\partial I / \partial V_{\mathrm{sd}}-V_{\mathrm{NWGA}}-V_{\mathrm{sd}}$ plot of a device with $40 \mathrm{~nm}$ wide underlying Si NW and three highly regular top gate NWs (Figure S4), recorded at $4.3 \mathrm{~K}$. Coulomb blockade diamonds of similar sizes appear to arrange into groups of three, as marked out by dotted frames.

$10 \mathrm{meV}$ addition energy is reflected in the gate voltage domain by the distance between consecutive diamonds or the width of each diamond in $V_{\mathrm{g}}$, which is estimated to be $\Delta V_{\mathrm{g}} \sim 30 \mathrm{mV}$. The gate coupling factor can therefore be calculated as: $\alpha=$ $\Delta V_{\mathrm{sd}} / \Delta V_{\mathrm{g}}=C_{\mathrm{g}} / C=0.33$, where $C_{\mathrm{g}}$ and $C$ are the gate capacitance and total capacitance of each QD, respectively. This $\alpha$ value is close to those reported for systems with similar dimensions. $^{22}$

The gate capacitance $C_{\mathrm{g}}$ of each dot can be calculated from $\Delta V_{\mathrm{g}}$ assuming charging energy $U=e^{2} / C$ is the dominant component of $E_{\text {add }}$ as compared to the other component $\Delta E$, the single-particle energy difference between consecutive quantized energy levels of the QD. This assumption should be valid for the dimension discussed here and leads to: ${ }^{22}$

$$
C_{\mathrm{g}}=\alpha C=\alpha \frac{e^{2}}{U} \sim \alpha \frac{e^{2}}{E_{\mathrm{add}}}=\alpha \frac{e}{\Delta V_{\mathrm{sd}}}=\frac{e}{\Delta V_{\mathrm{g}}}=5.3 \mathrm{aF}
$$

This value is in good agreement with the gate capacitance calculated from the geometry of each $\mathrm{QD}, C_{\mathrm{g}}{ }^{\prime}=4.7 \mathrm{aF}$, indicating that the QDs in this system are really defined by the NWGA.
The QD size effect was examined by comparing the data reported above with the results obtained from devices with significantly smaller underlying SiNWs. Figure 6a shows the Coulomb blockade diagram of one of the devices shown in Figure $4 \mathrm{c}$, with a $\sim 17 \mathrm{~nm}$ wide underlying Si NW, and Figure $6 \mathrm{~b}$ shows the data on another device with a $\sim 20 \mathrm{~nm}$ wide underlying Si NW (micrograph shown in Figure S3 of the Supporting Information). Diamonds of similar sizes were obtained with a certain amount of overlapping structure for both devices, in agreement with the observed modest wandering of the NWGA (Figure 4c and Figure S3b). Note the change of the $\left(V_{\mathrm{sd}}\right)$ energy scale for these plots as compared with those of Figure 5 and Figure S1. The addition energy $E_{\text {add }}$ directly measured from the maximum in $V_{\text {sd }}$ for the conductance gap of the diamonds (Figure 6a) is $30-40 \mathrm{mV}$ for the device with $\sim 17$ $\mathrm{nm}$ wide underlying $\mathrm{Si} \mathrm{NW}$, more than double the value obtained on devices with $\sim 40 \mathrm{~nm}$ wide underlying Si NWs (10$15 \mathrm{mV})$. This is consistent with the formation of smaller QDs along the SNAP Si NWs. The addition energy for the device with a $\sim 20 \mathrm{~nm}$ wide underlying $\mathrm{Si}$ NW is estimated from Figure $6 \mathrm{~b}$ to be $25-30 \mathrm{mV}$, a little smaller than the $\sim 17 \mathrm{~nm}$ device 
and about twice that of the $\sim 40 \mathrm{~nm}$ devices, again in agreement with the size of each individual QD defined by the device geometry.

Grouping of Coulomb blockade diamonds resulting from the coupling of QDs was observed in a device with three evenly spaced top gate electrodes (Figure 7). A micrograph of the device is presented in the Supporting Information (Figure S4). Very little wandering of the NWGA is observed in the SiNW region of this device, and this allows for the formation of three QDs of nearly identical sizes that electronically couple with each other. In the $\partial I / \partial V_{\text {sd }}-V_{\text {NWGA }}-V_{\text {sd }}$ plot, Coulomb blockade diamonds of similar sizes appear to be arranged into groups of three, as marked out by dotted frames in Figure 7. The present findings are for a system under the influence the top NWGA. The structure seen, however, is reminiscent of previously reported results where a gate is imposed on each one of serially coupled QD systems, where three coupled QDs defined in a GaAs/AlGaAs two-dimensional electron gas gave rise to conductance peaks that were similarly arranged into groups of three at zero source-drain bias. ${ }^{14}$

\section{Results from Model Computations}

The quantitative purpose of the model is to simulate the results for the conductance, $\partial I / \partial V_{\text {sd }}$, as a function of the source drain voltage, $V_{\text {sd }}$, and the gate voltages, $V_{\text {NWGA }}$, as shown schematically in Figure 2 and for real data in Figures 5-7. To allow for nonlinear responses, we must include the effects of the voltages in the Hamiltonian and this means that each point in the plot requires a separate diagonalization of the Hamiltonian. In the results shown in Figures $5-7, V_{\text {sd }}$ is low and can likely be described by a linear approximation. Even so, the top gate architecture means that for each value of the gate voltage the electronic problem is different and needs to be solved anew. This is because the NWGA, as opposed to a back gate, has gaps between the electrodes. Si atoms under the gaps are not influenced while other $\mathrm{Si}$ atoms that are under the gate are. It is precisely this feature that allows a new electronic structure to emerge when the top gate voltage is increased. However, it also means that the quantitative generation of a realistic Coulomb diamond plot is necessarily computationally intensive.

A simple model and yet one that seems to capture the essential physics is a tight-binding (or Hückel ${ }^{55-57}$ ) approximation applied on the atomic level. For the linear dimension of the wire as used in the experiment (of Figure 5) with four top gates, there are about $330 \mathrm{Si}$ atoms spanning the source and drain contacts (60 atoms between a source or drain electrode to the first gate $=2 \times 60,4 \times 30$ atoms under the gates, and $3 \times 30$ atoms in the gaps between the gates). Even in the most simplistic model, every point in the Coulomb diamond plot requires diagonalizing a 330 by 330 Hamiltonian matrix. Such a minimal description requires the drastic approximation of only one valence orbital per atom, what chemists refer to as the $\pi$-electron approximation. There are four valence orbitals, an $s$ and three $p$ orbitals, of each $\mathrm{Si}$ atom and using four equivalent linear combinations of these orbitals, the $s p^{3}$ hybridization generates the tetrahedral packing of solid silicon. For $N \mathrm{Si}$ atoms, there will be $4 N$ orbitals and these are split by the tetrahedral packing into a valence band of $2 N$ orbitals, which are fully occupied, and a higher lying conduction band of empty $2 N$ orbitals. To mimic a onedimensional wire, we take one $p$ orbital per Si atom, an orbital perpendicular to the wire. The other electrons provide the bonding that gives rise to the skeleton that holds the wire. We couple the $p$ orbitals by an exchange coupling that is estimated from the effective mass in $p$ type doped $\mathrm{Si}$. Thereby, we describe a valence band with holes due to the doping. Otherwise, the concentration of the boron atom dopants is low enough that their effect is included only to the extent that in the wire very few orbitals will be unoccupied, meaning that there are a small number of holes in the valence band.

To mimic the role of the NWGA, the energies of those $\mathrm{Si}$ atoms that are under the NW gate electrodes are shifted down (or up, depending on the sign of the voltage). The tight-binding Hamiltonian written in the basis of Si atomic orbitals, one orbital per atom, is

$$
H=\sum_{i=1}^{N} \alpha_{i}|i\rangle\left\langle i\left|+\sum_{i=1}^{N-1} \beta\right| i\right\rangle\left\langle i+1\left|+\sum_{i=2}^{N} \beta\right| i\right\rangle\langle i-1|
$$

where $\alpha_{i}$ is the ionization energy of atom $i$ where the atoms are numbered consecutively across the source and drain electrodes so that the value of $i$ defines the location of the atom along the wire.

In eqs 1 and $2, \beta$ is the exchange integral between adjacent $\mathrm{Si}$ atoms. By equating two expressions for the quadratic dispersion relation at the bottom of the band, $\hbar^{2} k^{2} / 2 m_{\text {eff }}=$ $2 \beta a^{2} k^{2}$, and on the basis of an effective mass of 0.2 and a lattice constant of $a=10 \mathrm{au}$, we determine $\beta=0.34 \mathrm{eV}$. Unlike an array of QDs ${ }^{58,59}$ and consistent with the tight-binding approximation, the Hamiltonian in the atomic basis does not include any explicit charging energy. The charging energy will emerge from the spectrum of energy levels, see Figure 9 below, when the top gate voltage is large enough so that $\chi$ is not negligible.

Without an applied field, the atomic energies $\alpha_{i}$ are identical, so that due to the exchange coupling the molecular orbitals (MOs) will be completely delocalized. To remove the degeneracy of the $\alpha_{i}$ values, we include the role of $V_{\text {sd }}$ by making the $\alpha_{i}$ values vary monotonically such that the difference in energies between two adjacent atoms is $e V_{\mathrm{sd}} /(N+1)$. Because $V_{\mathrm{sd}}$ is low, this is a minor quantitative correction.

The energies of atoms along the gated $\mathrm{Si}$ wire fall into two classes, as shown in Figure 8 below. For the dimensions of the device used in the experiment of Figure 5, atoms 1-60 and other $\mathrm{Si}$ atoms that are under the gaps of the top gate electrodes have the energy $\alpha$ appropriate for a valence orbital of a $\mathrm{Si}$ atom, except that it is very slightly modified by $V_{\text {sd }}$. Atoms $61-90$ and the other $\mathrm{Si}$ atoms under the top gates have different energy. We designate it as $\alpha+\Delta \alpha$ where $\Delta \alpha$ is the shift due to the gate voltage and the value of $\Delta \alpha$ is the electronic charge times the gate voltage as seen by the dot. Also, for the atoms that are under the gate, there is the additional very slight monotonic variation in $\alpha$ due to $V_{\mathrm{sd}}$. We keep this variation in the Hamiltonian, but because over much of the range $e V_{\mathrm{sd}} /(N+1)$ $\ll \Delta \alpha$, we make the qualitative discussion as if $\Delta \alpha$ is the entire difference in the atomic term values. We also keep $V_{\text {sd }}$ in the Hamiltonian because the source-drain induced shift splits the degeneracy of the levels of adjacent atoms. This split makes for a systematic variation in $\Delta \alpha$. In addition, there are random fluctuations because experimentally all of the top gates are not exactly the same but we expect these fluctuations to be small with respect to the mean effect of $V_{\text {NWGA }}$.

The Hamiltonian (eq 2) has the same dimensionless coupling parameter $\chi$ as for arrays of compressed colloidal QDs, ${ }^{12,50}$ but in the present problem, the magnitude of $\Delta \alpha$ can be varied by varying $V_{\text {NWGA }}$. One can thereby tune the electronic structure. This would be far harder to do with colloidal QDs where $\Delta \alpha$ is due to size variations of the dots, ${ }^{10,60}$ for an experiment with a different value of $\Delta \alpha$ requires a new synthesis of the dots, 

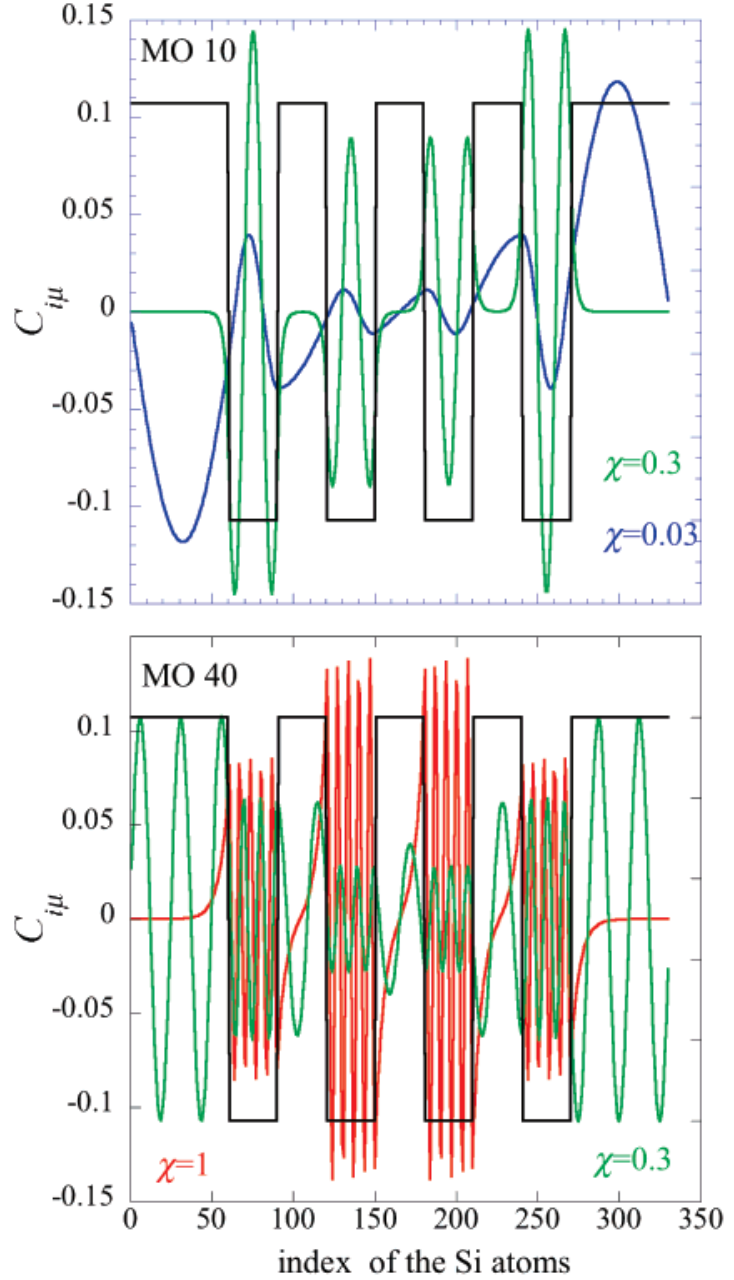

Figure 8. Amplitude coefficients, $C_{i \mu}$ for the expansion of the MO $\mu$ $=10$ (top) and $\mu=40$ (bottom) on the Si atoms, indexed by $i$, plotted vs $i$, and computed for increasing values of the $\chi$ parameter. As can be seen from Figure 9 below, for $\chi=0.03$, blue, almost all of the MOs are delocalized, so that both MO 10 and MO 40 are delocalized $(\chi=$ 0.03 not shown for MO 40). At $\chi=0.3$, green, MO 40 is still delocalized, but the lower energy MO 10 is already localized. At $\chi=$ 1 , red, MO 10 remains localized and its wave function, not shown, is hardly different from that at $\chi=0.3$. At $\chi=1$, the higher energy $\mathrm{MO}$ 40 is also localized. The profile of the energies of the two kinds of Si atoms as imposed by the top gates is shown in black.

reassembling a compressed array, etc. Particularly, near a quantum phase transition where the electrical properties are very sensitive to the compression of the array, the method discussed here is clearly superior for controlling variations in $\Delta \alpha$.

The ability to control $\Delta \alpha$ suggests that we can experimentally tune the conductivity. In the strong localization limit, $V_{\mathrm{NWGA}}$ is high and so is $\Delta \alpha$. This leads to $\chi>10$ so that the wire ceases to conduct. This is the familiar classical effect of the gate: A sufficiently high voltage can turn the conduction off. For incoherent conduction, one says that, depending on its sign, the gate has driven away all of the holes (or all of the electrons, depending on the doping). This traditional gating effect is present in that our quantum mechanical description does show that conduction ceases at very high gate voltage where $\chi>10$. However, as we shall see in the discussion followed, we also have the very interesting intermediate coupling, $10>\chi>1$, regime where the current is not a monotonic function of $V_{\text {NWGA }}$ and a quantum phase transition is observed as a discontinuity in the dependence of the energy on the number of electrons.

Figure 8 shows two panels of wave functions plotted vs the

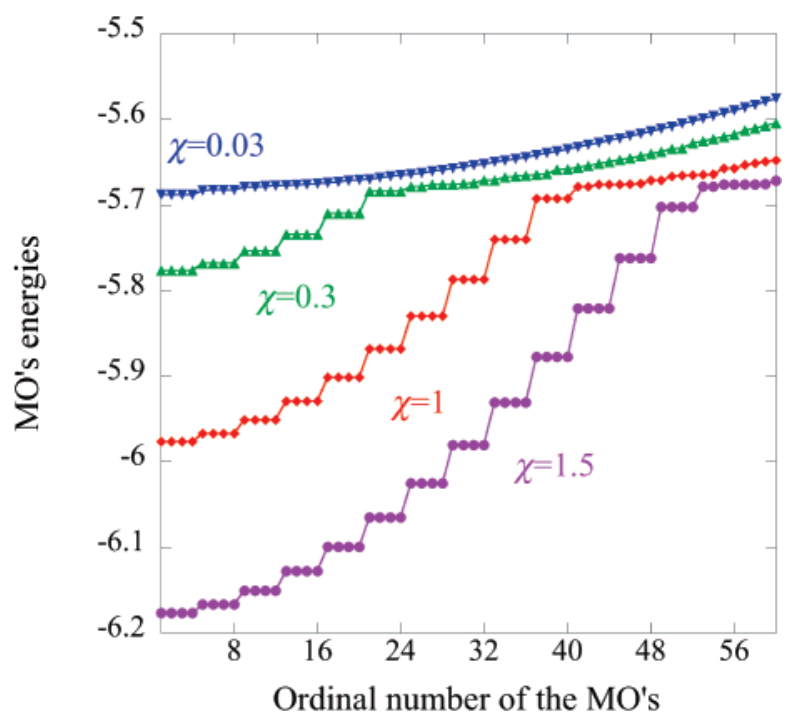

Figure 9. MO's energies plotted as a function of the ordinal number for a monatomic $\mathrm{Si}$ wire with four top gates. The quantum well structure emerging from imposing a nonzero voltage on the four top gates is clearly seen as well as the transition from a discrete level structure to a band structure as the energy increases. The values of the parameter $\chi$ of the tight-binding Hamiltonian are indicated in the figure. The computations are for a fixed value of $\beta$ and increasing values of $\delta \alpha$. For larger values of $\delta \alpha$, there are more levels in the wells. Note that the smooth curve fitted to the staircase dependence of the energies of the quantum number is quadratic, as expected for a particle in a well. Note further that past the quantum phase transition, when the dependence of the energy on the quantum number smoothes out, the "length" parameter of the well significantly increases. This reflects that past the transition the electron is no longer confined to a region under a top electrode but is delocalized along the entire wire.

number of the $\mathrm{Si}$ atom that corresponds to its position along the wire. The top panel is for a wave function with an energy near the bottom of the band (the 10th MO starting from the bottom) while the bottom panel is for a wave function somewhat higher in energy (MO 40). The computation is for a device with four top gates, and results are shown for increasing values of $\Delta \alpha: 0.01,0.1$ and $0.3 \mathrm{eV}$, two values shown in each panel. The corresponding values of the $\chi$ parameter, $0.03,0.3$, and 1 , are indicated in the pane. The transition from a localized to a delocalized character can occur as a function of two parameters: first, as $V_{\mathrm{NWGA}}$ increases, the wave function becomes localized within the wells created by the top gates. For $\chi=1$ (large $V_{\mathrm{NWGA}}$ ), the two wave functions are completely localized at those $\mathrm{Si}$ atoms that are under the gates. In that case, the orbital does not contribute to the conductivity because it has no weight on the $\mathrm{Si}$ atoms near the source or drain electrodes. When $\chi$ decreases, the higher energy MO (MO 40) first becomes delocalized, see plot, for $\chi=0.3$, but the lower energy orbital, MO10, is still localized. At $\chi=0.03$, both MOs are delocalized.

The primary point of the model computation is that localized sets of states emerge when the gate voltage $V_{\text {NWGA }}$, and therefore $\Delta \alpha, \chi=\Delta \alpha / \beta$, is increased. Figure 8 also illustrates a second route to the transition from localized to delocalized electronic structure. This theoretical route corresponds to the gate voltage $V_{\text {NWGA }}$ remaining constant in value so $\Delta \alpha$ and hence $\chi$ are held constant. What is varied is the range of energy of the states that conduct charge between the two electrodes. To realize this experimentally, one has several options. One is to apply a back gate voltage. This will alter the range of states that are within the Fermi window defined by the two electrodes. For example, at $\chi=0.3$, MO 10 is localized but MO 40 is delocalized throughout the wire. Another option is to increase $V_{\text {sd. }}$ This 
broadens the Fermi window and hence allows additional orbitals to have their energies within the window.

What controls the energy of the transition from a localized to a delocalized wave function is the value of the gate voltage or, more directly, the value of $\Delta \alpha$ that it implies. As an example, Figure 9 shows the energies of the MOs that make the band as a function of their ordinal number for increasing values of $\Delta \alpha$ (same values as in Figure 8). For $\chi \geq 1$, one clearly sees the four degenerate orbitals that are the four QDs that emerge from the computation without having been put in. As the ordinal quantum number increases, or equivalently, the energy goes up, the QDs are first seen to have a discrete level structure, that is, a finite charging energy (the incremental energy needed to add the fifth, ninth, etc. electrons). Then, and suddenly, the plot of energy vs quantum number changes slope and the discrete QDs are replaced by a band structure. The higher the value of $\Delta \alpha$ is, the higher the energy at which this transition occurs. At low $\Delta \alpha$, there are no QDs but only a band structure (see $\chi=0.03$ in Figure 9 where this limit is almost reached). At high $\Delta \alpha$, there is no band but only QDs.

Our system is finite, and so the energy is not strictly a continuous function of the number of electrons. To identify a phase transition as a discontinuity in the dependence of the energy on the number of electrons, one needs to pass a continuous smooth line through a staircase function shown in Figure 9. Then, it is seen that the curve changes slope abruptly after 4 or 8 or 12, etc. electrons have been added. For example, for the case $\chi=0.3$, the discontinuity occurs after 24 electrons have been added.

Popular texts ${ }^{42}$ dealing with emergent phenomena use such words as feedback or adaptive learning to explain the emergence of a new structure. To us, it seems that the term more suitable for the phenomena shown in Figures 8 and 9 is self-organization: It is the interaction between the subunits that gives rise to the emerging new structure.

A central issue is that all of the above features reflect what is observed in the experiment. Capturing this essential physics within our model is important for two reasons. First, it is our eventual intent to use the response of such a device for the purpose of implementing logic. For this, we need to understand the physics. Second, we want to make sure that we have correctly described the basic science. Our theory makes three cardinal approximations that need to be checked. First, we use a picture of coherent charge propagation through the wire to compute the Coulomb map shown (Figure 10). This is an assumption that we have previously used for arrays of metallic QDs, ${ }^{12}$ and we use the same formalism for computing the current here. The coherent approximation has also been used for short silicon wires. ${ }^{22}$ Still, it needs to be further checked, also with respect to the assumption used in the model that each gate is of the same width and that the spacings between the top gates are equal. We expect that this will take us to emerging dots that are of unequal size and coupling. As an intermediate check, we have used a Hubbard Hamiltonian of four coupled QDs, as in refs 46, 58, and 59, to reproduce the structure shown in Figure 10. A second approximation is that we describe the wire as a one-dimensional row of $\mathrm{Si}$ atoms. In actuality, the wire is a slab of silicon of finite width and thickness. Experimentally, we can fabricate and test thinner NWs, but any wire that we test will obviously have finite dimensions. Thus, why not do a band computation on a slab of silicon? This is because the influence of $V_{\text {NWGA }}$ within the source-drain axis of the NW leads to a distinct electronic structure and so requires diagonalizing the Hamiltonian for each value of the gate voltage. Including a

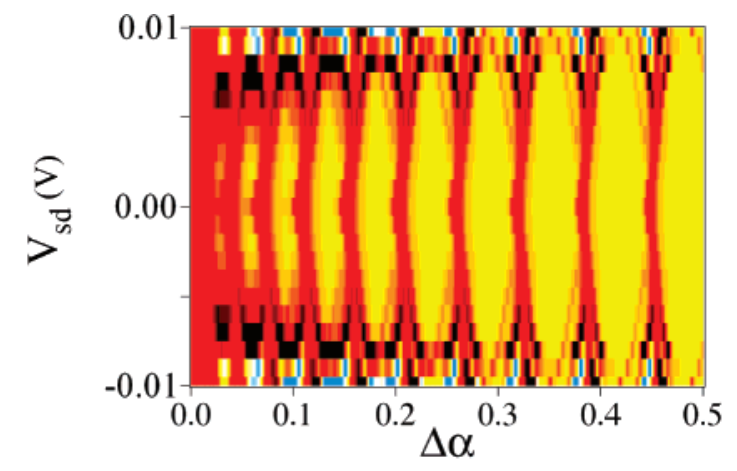

Figure 10. Computed Coulomb map $\left(\partial I / \partial V_{\mathrm{sd}}\right)$ for a $\mathrm{Si} \mathrm{NW}$ with four top gates. The abscissa $\delta \alpha$ is proportional to the voltage applied on the top gate, and the ordinate is the applied source-drain bias. Red represents high conductance, and yellow represents low conductance. One can see that as the top gate bias increases (high values of $\delta \alpha$ ), the device becomes insulating. Between each island, there is a narrow not fully resolved region of high conductance with more than one level. They correspond to the levels in the four wells (see Figure 9) but with their degeneracy lifted because of the source-drain voltage.

full band structure of a finite-sized Si NW into our calculations would be prohibitive. A third, but more minor approximation, is that we use an independent electron approximation for the valence band of $\mathrm{Si}$. We expect that electron correlation will change the results presented here but only in their details. Finally, we represent the doping as holes in the valence band of unperturbed $\mathrm{Si}$. The numbers of dopant atoms used in the experiment imply that this last approximation should be fine. Additional validation of the model will be forthcoming when it is possible to independently vary the voltage on the individual NWs within the gate array.

\section{Conclusions}

The experiment and computations provide compelling evidence that linear array of QDs can be imposed and tuned by an appropriately designed external gate voltage. Even with stateof-the-art fabrication methods, the size distribution of the QDs formed is going to be a fairly large fraction of the average size of the QDs, and we find that this disorder is reflected in the transport data. Nevertheless, both the disorder of the array and the coupling of the QDs are reflected in the fine structure of the Coulomb diamond map. The same maps can be computed from a model Hamiltonian that contains no explicit reference to QDs or their capacitance. The gate capacitance of each QD calculated from the experimental diamond diagrams is in agreement with the gate capacitance calculated from the geometry of each QD that is defined by a top NW gate electrode. Our interpretation is that the dot structure emerges due to the resonance interaction between the $\mathrm{Si}$ atoms that is disrupted when a voltage shifts the levels of those atoms that are under the top gate but not of those atoms that are under the gap between the gate electrodes. The parameter measuring the extent of off-resonance is $\chi=\Delta \alpha / \beta$ where $\Delta \alpha$ is the shift in the atomic term values as induced by the gate voltage, see Figure 8 , and $\beta$ is the exchange coupling between neighboring Si atoms. A dot structure, meaning at least one localized level under each gate, requires a threshold value of $\chi$; see Figure 9. When $\chi$ is large, the wave functions are so localized that conduction ceases. However, at lower values of $\chi$, there are clear discrete level structure effects and a transition, as a function of energy, between localized and delocalized states. The ultimate validation of this model will be provided by experiments that are in progress where the voltage on different gate electrodes is 
independently varied. It will be interesting, for example, to explore the extent to which order can be "electronically" imposed upon the system via control over the individual gate electrodes. It will also be interesting to simultaneously impose a back gate voltage and thereby tune the levels of all atoms up or down together. We have recently reported on a fabrication procedure that should allow us to utilize a bottom metal gate, in addition to the top NWGA. ${ }^{61}$

Acknowledgment. This article is dedicated to the memory of Richard E. Smalley. Rick was a spectacular scientist, an inspirational teacher, a visionary leader, and a wonderful friend. The experiment-theory collaboration is supported by the U.S.Israel Binational Science Foundation, BSF, Jerusalem, Israel. J.R.H. acknowledges primary support from the Department of Energy (DOE) and partial support from the MARCO Center for Advanced Structures and Devices. F.R. is Directeur de Recherches, FNRS, Belgium. R.D.L. and F.R. thank the EC FET-Open STREP project MOLDYNLOGIC.

Supporting Information Available: Details of device fabrication and Figures $\mathrm{S} 1-\mathrm{S} 4$. This material is available free of charge via the Internet at http://pubs.acs.org.

\section{References and Notes}

(1) Kastner, M. A. Phys. Today 1993, 46, 24-31.

(2) Ashoori, R. C. Nature 1996, 379, 413-419.

(3) Grabert, H., Devoret, M. H., Eds. Single Charge Tunneling: Coulomb Blockade Phenomena in Nanostructures; Plenum: New York, 1992.

(4) Kouwenhoven, L. P.; Austing, D. G.; Tarucha, S. Rep. Prog. Phys. 2001, 64, 701-736

(5) Murray, C. B.; Kagan, C. R.; Bawendi, M. G. Annu. Rev. Mater. Sci. 2000, 30, 545-610.

(6) Burda, C.; Chen, X. B.; Narayanan, R.; El-Sayed, M. A. Chem. Rev. 2005, 105, 1025-1102.

(7) Guo, L. J.; Leobandung, E.; Chou, S. Y. Science 1997, 275, 649651.

(8) Likharev, K. K. Proc. IEEE 1999, 87, 606-632.

(9) Collier, C. P.; Saykally, R. J.; Shiang, J. J.; Henrichs, S. E.; Heath, J. R. Science 1997, 277, 1978-1981.

(10) Beverly, K. C.; Sample, J. L.; Sampaio, J. F.; Remacle, F.; Heath,

J. R.; Levine, R. D. Proc. Natl. Acad. Sci. U.S.A. 2002, 99, 6456-6459.

(11) Remacle, F.; Beverly, K. C.; Heath, J. R.; Levine, R. D. J. Phys. Chem. B 2002, 106, 4116-4122.

(12) Remacle, F.; Beverly, K. C.; Heath, J. R.; Levine, R. D. J. Phys. Chem. B 2003, 107, 13892-13901.

(13) Duruoz, C. I.; Clarke, R. M.; Marcus, C. M.; Harris, J. S. Phys. Rev. Lett. 1995, 74, 3237-3240.

(14) Waugh, F. R.; Berry, M. J.; Mar, D. J.; Westervelt, R. M.; Campman, K. L.; Gossard, A. C. Phys. Rev. Lett. 1995, 75, 705-708.

(15) Waugh, F. R.; Berry, M. J.; Crouch, C. H.; Livermore, C.; Mar, D. J.; Westervelt, R. M.; Campman, K. L.; Gossard, A. C. Phys. Rev. B 1996, $53,1413-1420$

(16) Gaudreau, L.; Studenikin, S. A.; Sachrajda, A. S.; Zawadzki, P.; Kam, A.; Lapointe, J.; Korkusinski, M.; Hawrylak, P. Phys. Rev. Lett. 2006, 97, 036807.

(17) Livermore, C.; Crouch, C. H.; Westervelt, R. M.; Campman, K. L.; Gossard, A. C. Science 1996, 274, 1332-1335.

(18) van der Wiel, W. G.; De Franceschi, S.; Elzerman, J. M.; Fujisawa, T.; Tarucha, S.; Kouwenhoven, L. P. Rev. Mod. Phys. 2003, 75, 1-22.

(19) Petta, J. R.; Johnson, A. C.; Taylor, J. M.; Laird, E. A.; Yacoby, A.; Lukin, M. D.; Marcus, C. M.; Hanson, M. P.; Gossard, A. C. Science 2005, 309, 2180-2184

(20) Tans, S. J.; Devoret, M. H.; Dai, H. J.; Thess, A.; Smalley, R. E.; Geerligs, L. J.; Dekker, C. Nature 1997, 386, 474-477.

(21) De Franceschi, S.; van Dam, J. A.; Bakkers, E.; Feiner, L. F.; Gurevich, L.; Kouwenhoven, L. P. Appl. Phys. Lett. 2003, 83, 344-346.
(22) Zhong, Z. H.; Fang, Y.; Lu, W.; Lieber, C. M. Nano Lett. 2005, 5 , $1143-1146$

(23) Bockrath, M.; Liang, W. J.; Bozovic, D.; Hafner, J. H.; Lieber, C. M.; Tinkham, M.; Park, H. K. Science 2001, 291, 283-285.

(24) Mason, N.; Biercuk, M. J.; Marcus, C. M. Science 2004, 303, 655658

(25) Postma, H. W. C.; Teepen, T.; Yao, Z.; Grifoni, M.; Dekker, C. Science 2001, 293, 76-79.

(26) Bjork, M. T.; Thelander, C.; Hansen, A. E.; Jensen, L. E.; Larsson,

M. W.; Wallenberg, L. R.; Samuelson, L. Nano Lett. 2004, 4, 1621-1625.

(27) Yang, C.; Zhong, Z. H.; Lieber, C. M. Science 2005, 310, 13041307.

(28) Fasth, C.; Fuhrer, A.; Bjork, M. T.; Samuelson, L. Nano Lett. 2005, 5, 1487-1490.

(29) Biercuk, M. J.; Garaj, S.; Mason, N.; Chow, J. M.; Marcus, C. M. Nano Lett. 2005, 5, 1267-1271.

(30) Fujiwara, A.; Inokawa, H.; Yamazaki, K.; Namatsu, H.; Takahashi, Y.; Zimmerman, N. M.; Martin, S. B. Appl. Phys. Lett. 2006, 88, 053121.

(31) Takahashi, Y.; Ono, Y.; Fujiwara, A.; Inokawa, H. J. Phys.: Condens. Matter 2002, 14, R995-R1033.

(32) Smith, R. A.; Ahmed, H. J. Appl. Phys. 1997, 81, 2699-2703.

(33) Koester, T.; Goldschmidtboeing, F.; Hadam, B.; Stein, J.; Altmeyer, S.; Spangenberg, B.; Kurz, H.; Neumann, R.; Brunner, K.; Abstreiter, G. J. Vac. Sci. Technol. B 1998, 16, 3804-3807.

(34) Augke, R.; Eberhardt, W.; Strahle, S.; Prins, F. E.; Kern, D. P. Microelectron. Eng. 1999, 46, 141-144.

(35) Tilke, A.; Blick, R. H.; Lorenz, H.; Kotthaus, J. P. J. Appl. Phys. 2001, 89, 8159-8162.

(36) Tilke, A. T.; Simmel, F. C.; Lorenz, H.; Blick, R. H.; Kotthaus, J. P. Phys. Rev. B 2003, 68, 075311 .

(37) Hu, S. F.; Wong, W. Z.; Liu, S. S.; Wu, Y. C.; Sung, C. L.; Huang,

T. Y.; Yang, T. J. Adv. Mater. 2002, 14, 736-739.

(38) Melosh, N. A.; Boukai, A.; Diana, F.; Gerardot, B.; Badolato, A.; Petroff, P. M.; Heath, J. R. Science 2003, 300, 112-115.

(39) Beckman, R. A.; Johnston-Halperin, E.; Melosh, N. A.; Luo, Y ; Green, J. E.; Heath, J. R. J. Appl. Phys. 2004, 96, 5921-5923.

(40) Wang, D. W.; Sheriff, B. A.; Heath, J. R. Small 2006, 2, $1153-$ 1158

(41) Wang, D. W.; Sheriff, B. A.; Heath, J. R. Nano Lett. 2006, 6, 10961100

(42) Johnson, S. Emergence; Scribner: New York, 2002.

(43) Kubatkin, S.; Danilov, A.; Hjort, M.; Cornil, J.; Bredas, J. L.; StuhrHansen, N.; Hedegard, P.; Bjornholm, T. Nature 2003, 425, 698-701.

(44) Park, J.; Pasupathy, A. N.; Goldsmith, J. I.; Chang, C.; Yaish, Y.; Petta, J. R.; Rinkoski, M.; Sthena, J. P.; Abruna, H. D.; McEuen, P. L. Ralph, D. C. Nature 2002, 417, 722-725.

(45) Yu, H. B.; Luo, Y.; Beverly, K.; Stoddart, J. F.; Tseng, H. R.; Heath,

J. R. Angew. Chem., Int. Ed. 2003, 42, 5706-5711.

(46) Chen, G. L.; Klimeck, G.; Datta, S.; Chen, G. H.; Goddard, W. A. Phys. Rev. B 1994, 50, 8035-8038.

(47) McConnell, H. M. J. Chem. Phys. 1961, 35, 508-515.

(48) Anderson, P. W. Phys. Rev. 1959, 115, 2-13.

(49) Phillips, P. Annu. Rev. Phys. Chem. 1993, 44, 115-144.

(50) Remacle, F.; Levine, R. D. J. Phys. Chem. B 2001, 105, $2153-$ 2162.

(51) Sample, J. L.; Beverly, K. C.; Chaudhari, P. R.; Remacle, F.; Heath, J. R.; Levine, R. D. Adv. Mater. 2002, 14, 124-128.

(52) Beckman, R.; Johnston-Halperin, E.; Luo, Y.; Green, J. E.; Heath,

J. R. Science 2005, 310, 465-468.

(53) Hollenberg, L. C. L.; Greetree, A. D.; Fowler, A. G.; Wellard, C. J. Phys. Rev. B 2006, 74, 045311.

(54) Greentree, A. D.; Cole, J. H.; Hamilton, A. R.; Hollenberg, L. C. L. Phys. Rev. B 2004, 70, 235317.

(55) Hoffmann, R. Solids and Surfaces; VCH: New York, 1988.

(56) Schatz, G. C.; Ratner, M. A. Quantum Mechanics in Chemistry; Dover: New York, 2001

(57) Zallen, R. The Physics of Amorphous Solids; Wiley: New York, 1983

(58) Remacle, F.; Levine, R. D. J. Am. Chem. Soc. 2000, 122, 40844091

(59) Remacle, F.; Levine, R. D. ChemPhysChem 2001, 2, 20-36.

(60) Beverly, K. C.; Sampaio, J. F.; Heath, J. R. J. Phys. Chem. B 2002 106, 2131-2135

(61) McAlpine, M. C.; Ahmad, H.; Wang, D.; Heath, J. R. Nat. Mater. 2007, 6, 379-384. 